\title{
High accuracy indirect optical manipulation of live cells with functionalized microtools
}

\author{
Gaszton Vizsnyiczai ${ }^{\dagger}$, Badri L. Aekbote ${ }^{\dagger \dagger}$, András Buzás, István Grexa, Pál Ormos, Lóránd \\ Kelemen* \\ Biological research Centre of the Hungarian Academy of Sciences, Institute of Biophysics, \\ Temesvári krt. 62, Szeged, Hungary, H-6726 \\ †Present address: NANOTEC-CNR Istituto di Nanotecnologia, Dipartimento di Fisica, Università di Roma "La \\ Sapienza", p. le A. Moro, 2, 00185 Roma, Italy \\ ${ }^{\dagger}$ Present address: Center for Soft and Living Matter, Ulsan National Institute of Science and Technology, 50 UNIST- \\ gill, Ulju-gun, Ulsan 44919, Republic of Korea (South Korea)
}

\begin{abstract}
Optical micro manipulation of live cells has been extensively used to study a wide range of cellular phenomena with relevance in basic research or in diagnostics. The approaches span from manipulation of many cells for high throughput measurement or sorting, to more elaborated studies of intracellular events on trapped single cells when coupled with modern imaging techniques. In case of direct cell trapping the damaging effects of light-cell interaction must be minimized, for instance with the choice of proper laser wavelength. Microbeads have already been used for trapping cells indirectly thereby reducing the irradiation damage and increasing trapping efficiency with their high refractive index contrast. We show here that such intermediate objects can be tailor-made for indirect cell trapping to further increase cell-to-focal spot distance while maintaining their free and fast maneuverability. Carefully designed structures were produced with two-photon polymerization with shapes optimized for effective manipulation and cell attachment. Functionalization of the microstructures is also presented that enables cell attachment to them within a few seconds with strength much higher that the optical forces. Fast cell actuation in 6 degrees of freedom is demonstrated with the outlook to possible applications in cell imaging.
\end{abstract}

Keywords: two-photon polymerization, optical micromanipulation, single cell trapping, fluorescence microscopy, holographic optical tweezers, functionalization

\section{INTRODUCTION}

Optical manipulation of live cell is an important task in many fields of biophysics from studying cell mechanics ${ }^{[1],[2]}$ to modifying cell growth ${ }^{[3],}{ }^{[4]}$. Optical tweezers, using tightly focused laser beams are often used to relocate, probe or deform cells without the physical contact of a hard object. However, the use of optical tweezers to directly trap cells carries the danger of its damage by the high intensity beams ${ }^{[5]}$. Although there are ways to circumvent this problem with the choice of wavelength and laser power ${ }^{[6], ~}{ }^{[7]}$, the use of intermediate objects for indirect cell trapping is a common practice now. Cell-attached microbeads have long been used, for their ease of use and well-characterized procedures. Applications include the stretching of red blood cells ${ }^{[8]}$ or cell stiffness measurement ${ }^{[9]}$. Their main disadvantage is the lack of rotational motion and that the manipulation beam is still close to the sensitive cell. In the recent years the application of extended objects in indirect optical manipulation experiments is becoming more and more popular ${ }^{[10]}$ owed to the possibility of the total 6 degrees of freedom motion. Their optimal trapping conditions and behavior has been extensively studied ${ }^{[11],[12]}$, and a few applications have also surfaced ${ }^{[13],[14],[15]}$. In this paper we introduce precise and fast indirect cell manipulation with extended intermediate object that is actuated by a holographic optical tweezer (HOT) and which is capable of fast 6 degrees of freedom motion. The structures were made with two-photon polymerization, and their surface was functionalized to facilitate fast and reliable cell attachment. The stability of the trapped cell using two types of structures is compared, and stable motion with 6 degrees of freedom is demonstrated. Finally, we used cell staining to visualize organelles of the trapped cell during actuation to demonstrate the possibility of using the method for live cell imaging.

* email: kelemen.lorand@brc.mta.hu, phone: 36-62-599-600 x419

Optical Trapping and Optical Micromanipulation XIII, edited by Kishan Dholakia, Gabriel C. Spalding, Proc. of SPIE Vol. 9922, 992216 · C 2016 SPIE · CCC code: 0277-786X/16/\$18 · doi: 10.1117/12.2237601 


\section{METHODS}

\subsection{Design and preparation of cell manipulators}

In our earlier paper we introduced a microtool for indirect trapping consisting of four spheroids and a flat attachment plate ${ }^{[16]}$. The four spheroids were trapped by a static HOT holding the cell-structure couple while the microfluidic environment was translated along one of the three spatial directions. In this design (Fig. 1a) the four spheroids and the attachment plate were positioned onto a common plane. This particular arrangement is useful when the cell is translated without lifting from the surface, only sliding on it. However, when the structure is lifted from it, the whole structure tilts such a way that the cell moves downward (Fig. 1, dashed contours). It is simply due to gravity, since the density of a cell $\left(1.05-1.1^{[17],[18]}\right)$ is larger than that of the surrounding aqueous solution. From the geometry of the system it follows that this tilt, besides the lifting, results in an almost exclusively axial displacement of the trapping spheroids (displacement directions represented by red arrows on Fig. 1); lateral displacement is only due to Brownian fluctuation. Therefore during this tilt, the trapping force appears practically only in the axial direction. We observed in several occasions that the tilt was so considerable that the axial trapping force was not sufficient to keep the structure in the trap.

In order to improve the stability we designed a different type of cell manipulator to reduce the effect of the tilt, and compared it to the flat-type. The new structure has only three trapping spheroids, and a dish-like attachment plate. Most importantly, the attached cell is moved out from the plane of the three spheroids and it is situated above the center of the triangle of the spheroids. The structure can be rotated to such a direction where the plane of the three spheroids is parallel to the optical axis as illustrated on the lower structure of Fig. 1, while the cell is observable practically without any hindrance. When the cell is lifted in this position, the cell-structure couple can of course still tilt. However, the tilting in this case results in the lateral displacement of the spheroids (lateral red arrows on the lower structure of Fig. 1) giving rise to a lateral force component, having much larger trap stiffness than the axial one. We expect that this structure easily overcomes the tilting effect and provides stable cell trapping and manipulation. Additionally, the distance of the disk from the trapping spheres $(9 \mu \mathrm{m})$ is designed such that the high NA beam only minimally illuminates the cell, and the structure remains compact with short arm of leverage for the cell to tilt the structure; the attachment disk has a radius of curvature of $9 \mu \mathrm{m}$. We note here that since equal laser power is used in the trapping beams, the optical stress center (marked as black crosses) introduced by Simpson and co-workers ${ }^{[19]}$ can be regarded as a pivot point for the tilt.

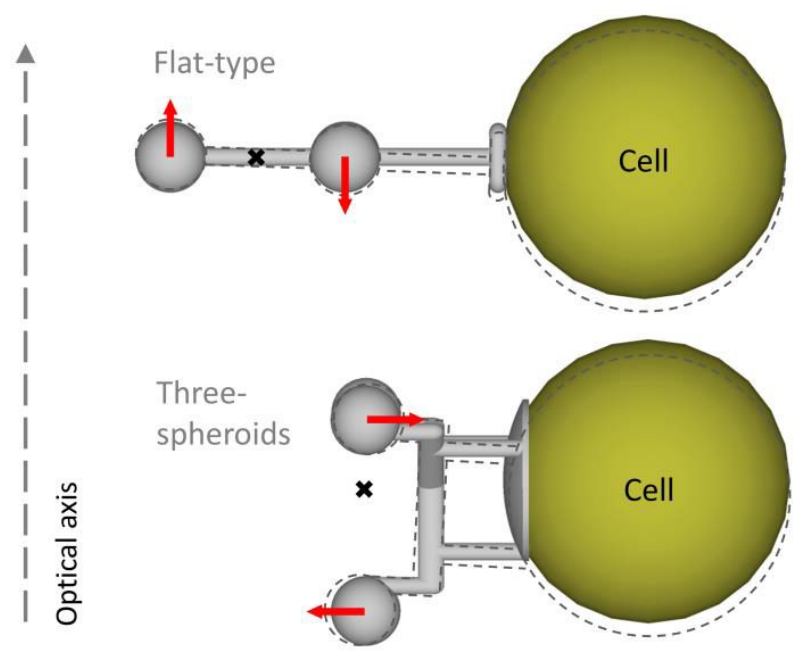

Figure 1.Schematic drawing of the two types of cell manipulator used in this work. The red arrows show the direction of displacement of the center of the trapping spheroids when the structure tilts upon lifting the cell with the trap; the dashed line shows the approximate position of the tilted structure.

We used two-photon polymerization to prepare these cell manipulator structures; the method is described in detail elsewhere. In short, polymerization occurred in a thin layer of SU8-2007 photoresist (Microchem, Germany) spin-coated onto a glass cover slide with the focused (Zeiss Apochromat, 100x oil objective, NA=1.25) beam of an ultrashort-pulsed laser (C-Fiber A, Menlo System, Germany, $785 \mathrm{~nm}$ wavelength, $100 \mathrm{fs}$ pulse length, $100 \mathrm{MHz}$ rep. rate). The sample was translated in 3D by a piezo scanner system (Physik Instrumente, Germany). The electronmicrograph of the prepared 3- 
spheroid structure is shown on Fig. 2. The cells (K562 cell line, provided by Ervin Welker, Institute of Biochemistry, Biological Research Centre) were attached to the structures exploiting the strong streptavidin-biotin linkage. For this the structures were functionalized to create a thin layer of streptavidin film on them, and the cells were treated with water soluble sulfo-NHS biotin that binds to their surface proteins, as described earlier ${ }^{[16]}$. In order to visualize cell nucleus, we selectively stained it by Hoechst 33342 fluorophore (abs.: $350 \mathrm{~nm}$, em.: $455 \mathrm{~nm}$ ) at low concentration that does not damage the cell, yet gives reliable fluorescent signal.

\subsection{Optical manipulation}

The optical manipulation experiments were performed on a Nikon Eclipse TI inverted microscope. The microscope was fitted with a holographic optical tweezers system, using a CW near infrared light source $(\lambda=1070 \mathrm{~nm}, \mathrm{P}=10 \mathrm{~W}$, THFL1P400-COL50, BKtel Photonics, France). The beam was coupled into the microscope with a high pass dichroic mirror (cut on wavelength: $\lambda=850 \mathrm{~nm}$ ). Their focusing was achieved by a NA=1.2 microscope objective $(60 \mathrm{X}$, water immersion, Olympus UPlanSApo). The microscope is also capable of observing the sample in wide-field fluorescence mode; we used filter set 49000 (exc.: 350nm, em.: 460nm, Chroma Technology Corp., USA) to visualize nucleus-stained cells. The holograms of the HOT system were displayed onto the reflective surface of a phase only spatial light modulator (SLM, PLUTO NIR, Holoeye, Germany). The holograms were calculated using an NVIDIA CUDA GPU as described in ${ }^{[20]}$, with the weighted Gerchberg-Saxton (GSW) algorithm ${ }^{[21]}$. We could calculate the 3 or 4 foci with 10 iterations easily within the $16.67 \mathrm{~ms}$ refresh cycle of the SLM, enabling real time, continuous and fast repositioning of the traps holding the cells indirectly. The total power contributing to the trapping beams was $270 \mathrm{~mW}$ at the objective's back aperture. A home-made user interface helped actuate the cell manipulator structures with $6 \mathrm{DoF}$ and control the fluorescence observation (EMCCD camera, Rolera EMC2, Qimaging, Canada).

The cell manipulation experiments were performed in HEPES buffer prepared from 5mM HEPES (4-(2Hydroxyethyl)piperazine-1-ethanesulfonic acid), $150 \mathrm{mM} \mathrm{NaCl}, 5.2 \mathrm{mM}, \mathrm{KCl}, 2.2 \mathrm{mM} \mathrm{CaCl}, 0.2 \mathrm{mM} \mathrm{MgCl}_{2} .6 \mathrm{H}_{2} \mathrm{O}, 6 \mathrm{mM}$ $\mathrm{NaHCO}_{3}$ and $2.8 \mathrm{mM}$ D-glucose. The streptavidin-coated and mechanically detached structures were injected into a microfluidic chamber, and the cells were injected subsequently. After trapping of a structure, it was pushed against a selected cell and within 5 seconds they were attached. Then, for the manipulation the indirectly trapped cells were lifted above the surface at least 10 micrometers to give enough free range for the entire structure. We used high framerate video microscopy (1000fps) to measure the stability of the cell-attached 3 -spheroid structure.

\section{RESULTS}
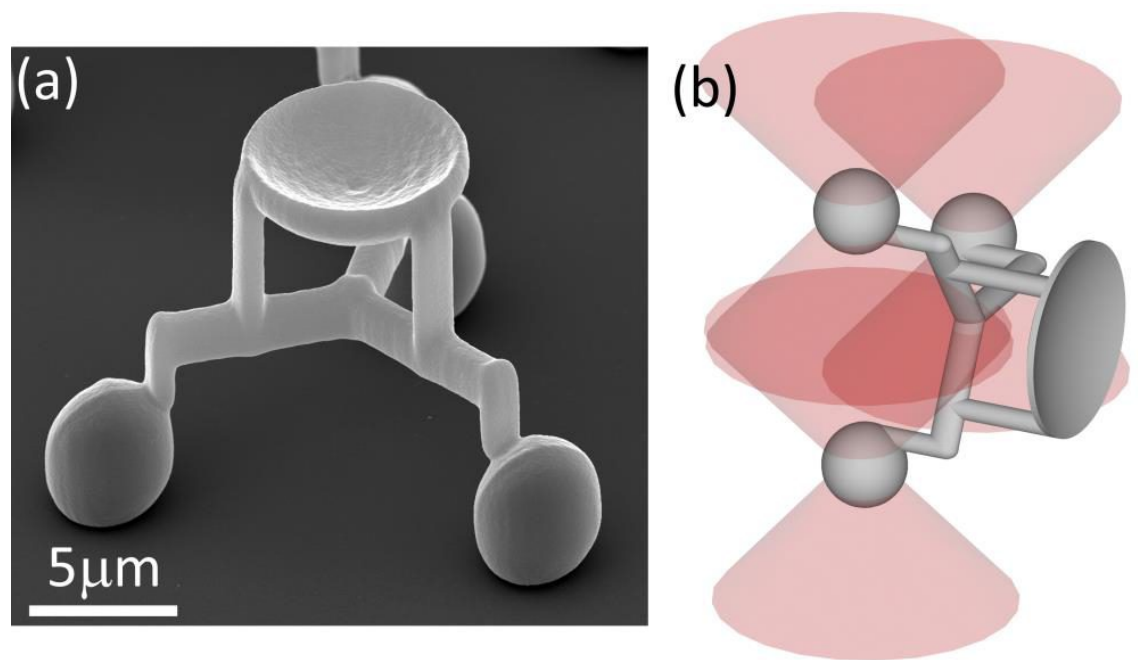

Figure 2.Electronmicrograph of a 3-sphere cell manipulator structure made of SU8 by two-photon polymerization (a). Schematic drawing of the 3 -sphere manipulator trapped by 3 focal spots of a holographic optical tweezers (b); pink cones represent the focused trapping beams.

In our earlier paper we introduced a complex indirect cell manipulator structure of the type shown on Fig. 3a. We demonstrated than the structure is capable of translating the attached cell in any spatial coordinates using a steady multi- 
beam optical tweezers. We also observed that when this indirectly trapped cell was lifted from the substrate, it often fell out of the trap. As we mentioned earlier, it is due to the fact that during tilt the trapping spheres displace parallel to the optical axis, in which direction the trapping stiffness is relatively small. We could determine the lateral escape force for this structure to be approximately $15-17 \mathrm{pN}(\sim 4 \mathrm{pN}$ for one spheroid) with a lateral translation experiment observing an escape velocity of $\sim 50 \mu \mathrm{m} / \mathrm{s}$; we used the Stokes equation to calculate the escape force regarding the cell as a sphere of $8 \mu \mathrm{m}$ radius and the four spheroids of the structure to have $2 \mu \mathrm{m}$ radii. Taking into account that the axial trap stiffness is several times lower than the lateral one ${ }^{[22],[12]}$, from the above escape force value we can estimate the axial escape force to be no more than $3-5 \mathrm{pN}(\sim 1 \mathrm{pN}$ for one spheroid) with the same trapping conditions. On the other hand, we can also estimate the torque, a cell exerts on the structure when tilted. A cell with $8 \mu \mathrm{m}$ radius and density of $1100 \mathrm{~kg} / \mathrm{m}^{3}$ exerts an approximately $2 \mathrm{pN}$ force with an arm of leverage of $18-20 \mu \mathrm{m}$. This torque is acting against the $\sim 4 \mathrm{pN}$ axial force with arms of leverage of $5 \mu \mathrm{m}$; we believe that it can be small enough not to be able to hold the structure in place when it tilts.


Figure 3.Image snapshots showing the rotation of an indirectly trapped cell with the holographic optical tweezers. The images of the cell rotated with the flat type manipulator (a) were taken with 2 s interval while the ones actuated with the 3 sphere manipulator (b) with 400ms interval (Video1). http://dx.doi.org/doi.number.goes.here

In the present experiments we extended the ways of motion by adding rotational capabilities to the HOT setup by translating the trapping laser beams by the SLM. We experimented rotating the flat-type structure along all three coordinates, which gave inconsequent results due to the geometry of the structure. These rotations could only be performed, of course, when the cell remained in the trap after lifting it. The rotation along an axis parallel to the optical axis (Fig 3a), that is, perpendicular to the plane of the structure could be performed without any problem: a total $360^{\circ}$ of rotation was possible. The speed of rotation, with the given trapping parameters was limited to about 20 degrees/s; above that the structure was often lost for the trap. Rotation around the long axis of the structure was limited to a range of a few tens of degrees: when the rotation angle approached 90 degrees, the rod connecting the two spheroids approached being parallel to the optical axis and trapping was not stable any more. Also, rotating the structure around its short axis sometimes resulted in losing it.

The three-spheroid manipulator performed much better in all the cell manipulation experiments. First we have to note that cell attachment to this type of structure is as fast as was found for the previous type ${ }^{[16]}$ : within seconds after the structure is pushed against the cell, they are permanently linked. The shape of the manipulator is designed such that the three spheres form a $16 \mu \mathrm{m}$ side length triangle; therefore, when the structure is rotated such that the spheres are above each other, the beam trapping the upper sphere is minimally disturbed by the lower sphere. It is compact enough that shorter arm of leverage is formed between the center of the triangle of the trapping spheres and that of the cell (15$17 \mu \mathrm{m})$. The attachment disk in the lateral orientation only minimally blocks the view of the cell while provides large and properly-shaped attachment surface. Most importantly, when the cell is held horizontally, the cell tilt is compensated only by the lateral trapping force.

The translation and rotation cell manipulation could be reliably performed with this microtool. We can directly compare its rotation around the axis parallel to the optical axis with that of the flat-type structure. The achievable speed is more than 3 times larger here: even at 65 degrees/s rotation the cell is held stably (see Fig. 3b). The overall length of the structure-cell pair is only about $27 \mu \mathrm{m}$, while it was around $40 \mu \mathrm{m}$ for the flat-type, which results in a reduced viscous resistance. The rotation around the two lateral axes also results in stable movement; it is especially easy to rotate around 
the common axis of the cell-structure couple. In this direction the rotation is limited by the speed of the hologram recalculation and not by the viscous drag.
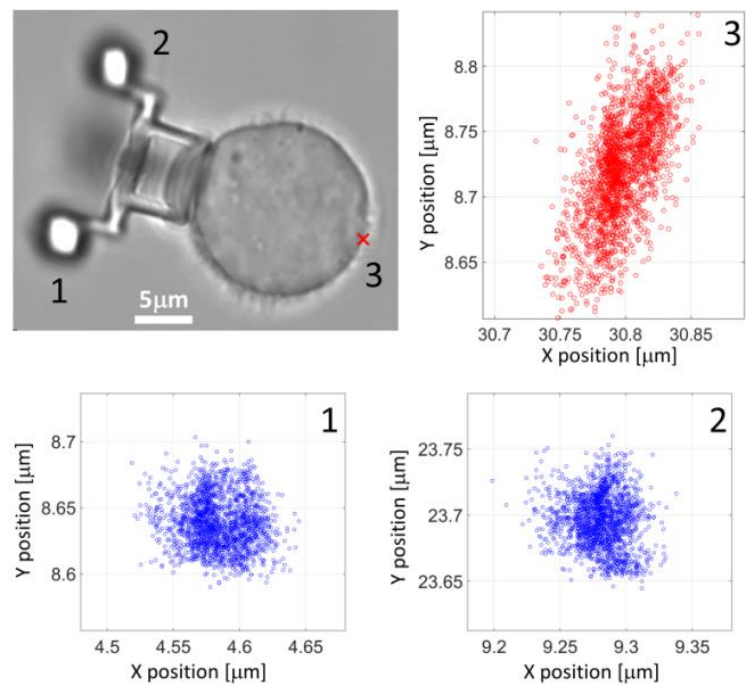

Figure 4.Position stability of an indirectly trapped cell shown on the optical microscope image. The center positions of the two visible spheroids in position 1 and 2 were measured by video microscopy and its distribution plotted with blue circles. The position of point 3 marked on the image by the red cross was calculated from the positions of spheres 1 and 2, and plotted as red circles.

The stability of the trapped structure-cell couple was also measured by holding it and measuring the positions of the trapping spheres. We found that the positions of spheres fluctuate with about 45nm FWHM (centers of sphere 1 and 2 on Fig.4) which results in an angular fluctuation of about 0.25 degrees. From this data we estimated the fluctuation of a point on the cell that situates $25 \mu \mathrm{m}$ away at the far end of it (red cross at position 3 on Fig.4), relative to the attached structure; this is the point that is displaced the most by the Brownian fluctuation. We found that the position of this point distributes with about 47nm FWHM along one axis and about 125nm FWHM along the other, perpendicular one.
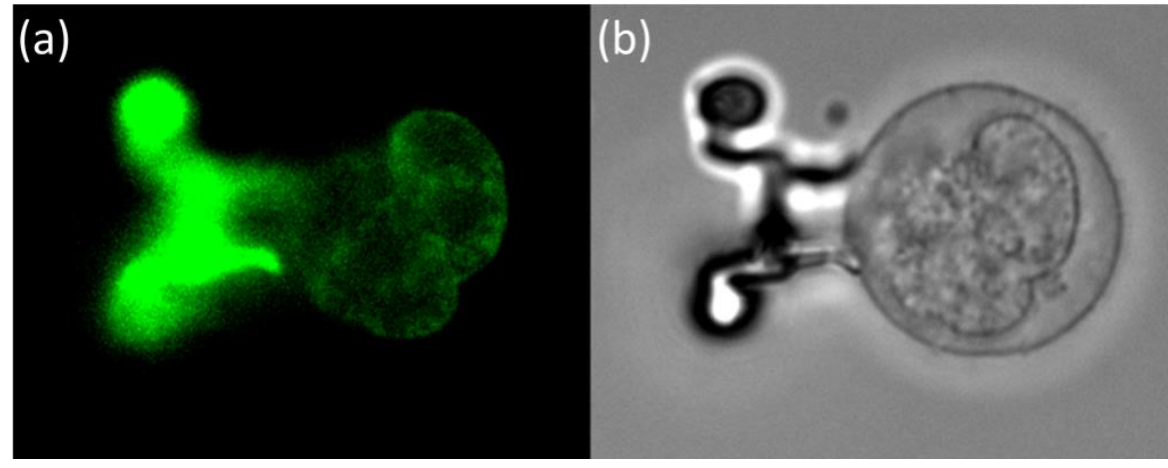

Figure 5.Fluorescence (a) and brigthfield (b) optical microscopic image of an indirectly trapped cell. The nucleus of the cell is stained selectively.

In order to demonstrate that indirectly trapping extends the potentials of single cell studies, we performed fluorescent observation of trapped cells. For this the cells nuclei were stained selectively with a dye that does not harm the cells at low concentration. The available fluorophores can be excited in the $400 \mathrm{~nm}$ wavelength range, where the SU8, the material of the manipulators unfortunately also absorbs and has strong autofluorescence. This did not prevent us from observing the shape of the nucleus of the indirectly trapped cell. Furthermore, when rotated the cell around, it was possible to observe it from multiple directions. This potential of the fast indirect cell manipulation can be greatly explored with imaging applications where the 3D structure of certain cell organelles can be determined. The precision of the cell positioning, quantified above, shows that the Brownian fluctuation does not significantly blur the image of a trapped cell. We emphasize that this is mainly due to the fact that the extended trapped object has large refractive index contrast relative to the surrounding medium of the indirectly trapped cell. 


\section{ACKNOWLEDGEMENTS}

The authors thank Dr. Péter Galajda (Institute of Biophysics, Biological Research Centre) for allowing the use of his fluorescent microscope system for the trapping experiments. The K562 cell line was kindly supported by Dr. Ervin Welker, Institute of Biochemistry, Biological Research Centre. This work was supported by funding received from the CONCERT-Japan Photonic Manufacturing Joint Call (in Hungary represented by the Hungarian Science Research Fund, grant no.: NN 114692). The work was also supported by the EU through the Economic Development and Innovation Operational Programme (GINOP-2.3.2-15-2016-00001). L.K. was supported by the Bolyai János Research Scholarship of the Hungarian Academy of Sciences.

\section{REFERENCES}

[1] Bronkhorst P. J. H., Streekstra G. J., Grimbergen J. et al., "A new method to study shape recovery of red blood cells using multiple optical trapping," Biophysical Journal, 69(5), 1666-1673 (1995).

[2] Rao S., Balint S., Cossins B. et al., "Raman Study of Mechanically Induced Oxygenation State Transition of Red Blood Cells Using Optical Tweezers," Biophysical Journal, 96(1), 209-216 (2009).

[3] Volpe G., Singh G. P., and Petrov D., "Dynamics of a growing cell in an optical trap," Applied Physics Letters, 88(23), 231106 (2006).

[4] Wu T., Mohanty S., Gomez-Godinez V. et al., "Neuronal growth cones respond to laser-induced axonal damage," Journal of the Royal Society Interface, 9(68), 535-547 (2012).

[5] Rasmussen M. B., Oddershede L. B., and Siegumfeldt H., "Optical tweezers cause physiological damage to Escherichia coli and Listeria bacteria," Applied and Environmental Microbiology, 74(8), 2441-2446 (2008).

[6] Liang H., Vu K. T., Krishnan P. et al., "Wavelength dependence of cell cloning efficiency after optical trapping," Biophysical Journal, 70(3), 1529-1533 (1996).

[7] Leitz G., Fallman E., Tuck S.et al., "Stress response in Caenorhabditis elegans caused by optical tweezers: Wavelength, power, and time dependence," Biophysical Journal, 82(4), 2224-2231 (2002).

[8] Dao M., Lim C. T., and Suresh S., "Mechanics of the human red blood cell deformed by optical tweezers," Journal of the Mechanics and Physics of Solids, 51(11), 2259-2280 (2003).

[9] Nawaz S., Sanchez P., Bodensiek K. et al., "Cell Visco-Elasticity Measured with AFM and Optical Trapping at Sub-Micrometer Deformations," Plos One, 7(9), e45297 (2012).

[10] Phillips D. B., Grieve J. A., Olof S. N. et al., "Surface imaging using holographic optical tweezers," Nanotechnology, 22(28), 285503 (2011).

[11] Grieve J. A., Phillips D. B., Hanna S. et al., "Non-spherical optically trapped probes: design, control, and applications," Proc. SPIE 8458, 84581 Y (2012).

[12] Phillips D. B., Simpson S. H., Grieve J. A. et al., "Force sensing with a shaped dielectric micro-tool," Epl, 99(5), 58004 (2012).

[13] Phillips D. B., Gibson G. M., Bowman R. et al., "An optically actuated surface scanning probe,” Opt Express, 20(28), 29679-93 (2012).

[14] Simons M., Pollard M. R., Hughes C. D. et al., "Directly interrogating single quantum dot labelled UvrA(2) molecules on DNA tightropes using an optically trapped nanoprobe," Scientific Reports, 5, 18486 (2015).

[15] Vizsnyiczai G., Lestyan T., Joniova J. et al., "Optically Trapped Surface-Enhanced Raman Probes Prepared by Silver Photoreduction to 3D Microstructures," Langmuir, 31(36), 10087-10093 (2015).

[16] Aekbote B. L., Fekete T., Jacak J. et al., "Surface-modified complex SU-8 microstructures for indirect optical manipulation of single cells," Biomedical Optics Express, 7(1), 45-56 (2016).

[17] Wolff D. A., and Pertoft H., "SEPARATION OF HELA CELLS BY COLLOIDAL SILICA DENSITY GRADIENT CENTRIFUGATION : I. Separation and Partial Synchrony of Mitotic Cells," The Journal of Cell Biology, 55(3), 579-585 (1972).

[18] Bryan A. K., Goranov A., Amon A. et al., "Measurement of mass, density, and volume during the cell cycle of yeast," Proceedings of the National Academy of Sciences of the United States of America, 107(3), 999-1004 (2010).

[19] Simpson S. H., and Hanna S., "Thermal motion of a holographically trapped SPM-like probe," Nanotechnology, 20(39), 395710 (2009). 
[20] Vizsnyiczai G., Kelemen L., and Ormos P., "Holographic multi-focus 3D two-photon polymerization with realtime calculated holograms," Optics Express, 22(20), 24217-24223 (2014).

[21] Di Leonardo R., Ianni F., and Ruocco G., "Computer generation of optimal holograms for optical trap arrays," Optics Express, 15(4), 1913-1922 (2007).

[22] Neuman K. C., and Block S. M., “Optical trapping,” Review of Scientific Instruments, 75(9), 2787-2809 (2004). 